\title{
Knowledge based Recommendation System in Semantic Web - A Survey
}

\author{
Ayesha Ameen \\ Professor \& Head of Department I.T. \\ Deccan College of Engineering and Technology \\ Darusalam, Hyderabad, Telangana, India
}

\begin{abstract}
Knowledge based recommendation systems use knowledge about users and products to make recommendations. Knowledge-based recommendations are not dependent on the rating, nor do they have to gather information about a particular user to give recommendations. Knowledge acquisition is the most important task for constructing knowledge-based recommendation system. Acquired knowledge must be represented in some structured machinereadable form, e.g., as ontology to support reasoning about what products meets the user's requirements. In Semantic Web, knowledge is represented in the form of ontology. Representation of knowledge in structured form of ontology in Semantic Web makes the application of knowledge based recommendations system on Semantic Web very easy, as there is no need to construct knowledge base from scratch. Performance of knowledge based recommendations systems can be enhanced by exploiting ontology reasoning characteristics. This paper explores different techniques used to generate knowledge-based recommendations highlighting the advantages of knowledge based recommendation system over other recommendation techniques.
\end{abstract}

\section{General Terms}

Recommendation systems

\section{Keywords}

Semantic Web, Ontologies, Reasoning, Knowledge base.

\section{INTRODUCTION}

World Wide Web has become a major source of information acquisition as it contains millions of documents related to any topic, which is of interest to users. Users often find it difficult to extract the relevant information from the documents returned as a result of the query posted on Web the reason behind this is, WWW contains documents which can be interpretable by only human but not by machine[1]. Recommendation system is used to solve this problem by generating personalized recommendations to Web users. Personalized recommendation in Web is no longer considered as an option but has become a necessity because of the movement from traditional physical stores of products or information to virtual stores of products and information [2]. As a result of this movement customers have a wide variety of options to choose from. Users can switch from one Website to another in virtual store; as many Websites offer the same type of services and products. It becomes difficult to retain customers in virtual store. Personalized recommendations help to solve the customer retention problem. Recommendation systems improve the trust of customer in business by building customer loyalty and one to one relationship by understanding the needs of each customer.

\section{RECOMMENDATION SYSTEM}

The aim of recommendation system is to generate meaningful recommendations to users for items that might be interesting to them. Recommendation systems have been widely used by information sources for personalizing their contents for the users [3]. In the context of Semantic Web, widely used recommendation approaches are content-based, collaborative filtering and knowledge-based [4]. Content based filtering approach analyses the contents of documents; collaborative filtering approaches are based on the opinion of group of users who have the same preferences; knowledge-based approaches utilize the knowledge in a structured form to produce personalized recommendations [5].

\subsection{Content-based filtering}

Content-based recommendation systems recommend items similar to the items a particular user has liked in the past [6]. Content based recommendation systems analyse items to identify those items that can be interesting to the users [7]. Recommendations are produced by matching up the attributes of the object or item with the user preferences or interest which is stored as attributes of user profile. Techniques used for content based recommendation systems differ in the way they analyse the items of documents or descriptions of items to build up user profile. The following paragraphs discuss some of the content-based filtering approaches used in Semantic Web

Ijntema et al. [8] used Concept Frequency-Inverse Document Frequency (CF-IDF) which is an adaptation of Term Frequency-Inverse Document Frequency (TF-IDF) with semantics for domain ontology to make recommendation for items. CF-IDF represents items in documents as weighted vectors of key concepts instead of terms. Weights are assigned accordingly. High weights are assigned to most discriminating features/preferences and low weights are assigned to less informative features/preferences, thereby avoiding noise term, which can pollute the recommendation output. User profile and items are represented in terms of CF-IDF. Recommendations are generated by comparing items with user profile using cosine similarity.

Bogdanov et al. [9] proposed a user model with input as explicit preferences from users instead of calculating CF-IDF as in the approach proposed by IJntema for making recommendations. Semantic descriptors are calculated for each input. Trained classifiers are used to obtain the class labels of each input represented as semantic descriptors. For each input, the classifier returns probability estimates of classes on which it was trained. Three approaches are used for producing recommendations. First two approaches represent the user model in term of vector and uses weighted Pearson correlation distance to calculate the distance between user model vectors and item vector. The first approach calculates the mean for the user model vector and recommends the item 
nearest to the mean vector. The second approach uses all the points in user model vector and recommends items that are close to any point in user model vector. The third approach represents user model as probability density preferences in semantic space. Recommendations are generated by using expectation maximization algorithm.

\subsection{Collaborative filtering recommendation systems}

Collaborative filtering recommendation creates groups of users according to their preferences and generates recommendation based on items liked by the other users belonging to the same group [10]. Collaborative filtering system uses similarity measures to compute the similarity between users and items recommended. Performance of collaborative filtering recommendation systems depend on different approaches used for similarity computation. Collaborative filtering recommendation system when applied to Semantic Web computes semantic similarity between items and users to produce personalized recommendations. Cold start problem can be reduced by recommending items that are semantically similar to the given item in Semantic Web. Item sparsity problem is reduced by mapping items and users to domain ontology in semantic collaborative filtering [11]. Some of the approaches used for collaborative filtering in Semantic Web have been discussed in the following paragraphs.

Lee et al. [12] used semantic collaborative filtering techniques based on personalized search Bayesian Belief network (pEBBN) to retrieve documents that have high semantic similarity to the given query. pEBBN uses domain knowledge to represent concepts in concept layer which represents the semantics of user preference queries and documents with their corresponding concepts. To personalize the search the first step is to find the implicit authority of document by collaborative filtering, which finds a set of user who has the same preferences as that of the user who has submitted the query. In the second step a score function which performs conceptual mapping along with the semantic similarities between like-minded users is computed on the documents accessed by like-minded users and the query submitted and the preferences of the user. Top-k documents are returned as a result of the search for each like-minded user.

Lee's approach computes semantic similarity between the documents and queries but does not focus on the semantic properties that are associated with items and users. Semantic properties can be utilized to gain more knowledge regarding items and users in recommendations system. Semantic properties are used in a new technique for collaborative filtering called property-based collaborative filtering (PBCF) in an approach proposed by López et al. [13]. PBCF represents the domain knowledge about items and users in the form of ontology to support reasoning about them in a formal way. PBCF separates users and their properties as well as items and their properties to build a matrix of values describing the influence of one item property of item on users with certain user property. This type of representation solves many problems that existed in traditional collaborative filtering recommendation systems.

The approach proposed by Lopez utilized semantic property information for generating recommendation, whereas the approach proposed by Fard et al. [14] computes semantic similarity between users to find nearest neighbours. Items are recommended to user based on the previous ratings of nearest neighbours. Semantic similarity method computes weighted sum between Relation Similarity, Taxonomy Similarity and Attribute Similarity which are the measures used for calculating semantic similarity between ontology concepts.

\section{KNOWLEDGE BASED RECOMMENDATION SYSTEMS}

Content based filtering and Collaborative filtering recommendations are suited for products that are purchased frequently, such as, books, news, and music. In case of items, such as, computers, cars, financial services, loans and apartments, which are not purchased, frequently, it becomes difficult to collect rating, and the user will not be satisfied with recommendations produced based on old item preferences. Therefore, using collaborative filtering and content-based recommendation techniques will not produce good quality recommendations. The challenges faced in the domain of items, which are not frequently purchased, are tackled by exploiting deep knowledge in the product domain and user's requirements by Knowledge-based recommendation techniques.

Knowledge-based recommendations are produced based on two approaches. The first approach is case based recommendations, which find products from the case base that are similar to the products described by the user's requirements. Past experiences that can be used to achieve the goals of the system are represented by cases. The second approach is constraint-based recommendation systems, which recommends items, based on explicit rules specified as constraints on knowledge base.

\subsection{Case-based recommendations}

Case-based recommendations are a form of content-based recommendation that uses organized knowledge in the form of cases to make recommendations to the users [15]. Case base consists of collection of previous problem or cases that have been solved. Each case consists of two parts. The first part describes the problem at hand and is called specification part. The second part describes the solution used to solve the problem and is called solution part. To solve new problems case whose specification matches up to the current problem are retrieved and the solution is adapted to suit the current problem. An approach for case based recommendation is discussed in the following paragraph.

Daramola et al. [16] proposed an approach based on ontological framework designed to produce knowledge-based recommendation to the users in tourism domain. Ontologies are developed for destination, restaurant and accommodation. User preferences are explicitly collected from the user and stored in the system. Past user preferences are stored as cases in case base. Architecture presented in this approach produces recommendations by matching current user preferences with the past cases and by performing semantic match between the ontological description of destinations, restaurant and accommodation stored in knowledge base of the system.

\subsection{Constraint-based recommendation}

Constraint based recommendation systems are also called Rule-based recommendation systems because they produce recommendation based on explicitly specified constraint or rules. The rules define the mapping between customer requirements and item features [17]. Recommender knowledge base consists of user requirements, item properties and rules. Items that satisfy the rules with respect to a given set of user requirement are generated as recommendation [18]. 
Rule based recommendation systems are suited for applications where user is ready to spend more time and effort to ensure that they get the best item recommended [19]. Mostly they are used where the user does not have sufficient knowledge about items domain like e-government, production systems, telecommunication switches, and financial services.

\section{Advantages of using Rule based recommendations}

Using Rule-based recommendations systems have many advantages, such as,

\section{Domain knowledge}

2. Rule-based recommendations are based on domain knowledge, which comprises all details about the domain along with the restrictions that exist in the domain.

\section{Result explanation}

4. Rule-based recommendation systems not only provide high quality recommendations, but also provide explanations for inclusion of item in recommendation list based on the combination of item properties, which lead to the selection of item.

Rule-based recommendation systems, when applied on Semantic Web can benefit from the underlying ontologies, which form the backbone of Semantic Web. Ontologies can be used to represent the domain knowledge and impose restrictions on the domain in a machine process able way. Ontologies support reasoning which determine new information automatically which is not explicitly specified in the knowledge base. Reasoning characteristic of ontology can be used to derive the explanation for the presence of items in the recommended list. Therefore, Semantic Web provides a better platform for implementing Rule-based recommendation system as it fulfils and extends the advantages of Rule-based recommendation systems [20].

\section{RULE BASED PERSONALIZED RECOMMENDATION IN SEMANTIC WEB}

Rule based Personalization systems produce recommendations, which meet certain requirements represented as rules. Rules form an integral part of Semantic Web-layered architecture. Logic and proof layers support rule layer in Semantic Web.

\subsection{Significance of rules in Semantic Web}

Rules provide a foundation for automated reasoning that supports intelligent exploitation and manipulation of information content [21]. Designing of rules to support the functioning of Semantic Web application was identified as a major design issue by Tim Berners Lee et al [22]. Listed below is the significant issue involved in the design of Semantic Web.

"Adding logic to Semantic Web \{the means to use rules to make inferences, choose courses of action and answer questions $\}$ is the task before the Semantic Web community at the moment."

Therefore, rules play a vital role in realizing the full potential of Semantic Web inference mechanism. Although ontologies support some basic form of Description Logic (DL) reasoning, they cannot support range of knowledge-based services that can be supported on Semantic Web [23]. To improve reasoning capabilities beyond OWL, rules are used in Semantic Web.

\subsection{Rules-based personalization}

Rule-based reasoning approaches can be used for personalization of Semantic Web by writing rules for implementing personalization logic. Rules represent knowledge with conditions in some domain of logic, such as, first order logic. A rule is defined as 'If-then' clause containing logical functions and operations, which can be expressed in a rule language. If-clause specifies the condition or premises and then- clause specifies the conclusion or action to be taken. If conditions are true in if-clause, then the conclusion or action will be carried out in the then-clause.

Reasoning based approaches for personalization in Semantic Web were first proposed by Antoniou et al. [24]. They categorized approaches into Monotonic, Non-monotonic, Evolution updates and events, and reasoning about actions.

Monotonic reasoning is static. The truth of statement does not change when new information is added and this type of reasoning is performed by DL reasoner which is based on Open World Assumption which allows easy integration of new information and the existing information truth value is not affected with the addition of new information.

Non-Monotonic is the reverse of Monotonic where adding of new information can affect truth value of existing information. Defeasible reasoning and Answer set programs are examples of non-monotonic reasoning systems. Defeasible reasoning is a rule-based approach which works with incomplete and inconsistent information [25]. It can represent facts, rules, and priorities among rules. Answer Set Programs are non-monotonic logic programs based on the Answer Set Semantics, which use extended logic programs for reasoning and problem solving by considering possible alternative scenarios [26]. Evolution updates and events represent dynamic aspects of personalization in Semantic Web [27]. This approach represents reactive behavior specifying actions to be taken according to the situation by writing rules. EventCondition-Action paradigm is used to represent the reactive behavior. An occurrence of a specific activity is an event, when an event occurs, a condition is checked; if condition is satisfied, an action is carried out.

Reasoning about actions and time is an example of temporal reasoning where reasoning is performed about the phenomenon that occurs in time using properties characterizing dynamic behavior with truth-value depending on changes occurring in the world.

$\mathrm{Mu}$ et al. [28] further refined the categorization proposed by Antoniou and categorized rule-based recommendation approaches into four major categories. Categories of Rule based Personalization approaches are mentioned are as follows.

1. Logical Languages

2. Event-Action-Rule

3. Expert systems

4. Rule based Inference Engine

The first category is based on extending the logical languages to provide personalized recommendations. The second category is the same as Evolution updates and events discussed in the preceding paragraph where rules are written for the occurrences of events and actions are carried out if 
conditions are true. The third category is Expert systems which consist of 'if-then' rules and knowledge base can be used for obtaining personalized recommendations by applying reasoning methods. The last category is of rule engine which are application software used to derive new knowledge from the existing knowledge. Personalization is done by applying rules for reasoning over data.

\subsubsection{Logical Languages}

Logical languages can be extended for supporting personalization in Semantic Web [29]. Description logic is a decidable fragment of first order predicate logic which is used to represent ontologies in Semantic Web [30]. Web Ontology language which is W3C recommended language for representing ontologies in Semantic Web is based on DL. DLs have strong inference mechanism. Therefore they can be used for Personalization by extending DL semantics. Some of the approaches used for rule-based personalization using logical languages are discussed in the following paragraphs.

Personalization performed by extending logical languages was demonstrated by $\mathrm{Mu}$ et al. OWL DL is extended by writing DL safe rule implementing personalization logic for application. A finite set of DL safe rules is called a logic program, which is at the higher conceptual level than the imperative programs consisting of if-then statements. DL safe rules are combination of OWL DL and function free Horn rules. Rules can be modified depending on the user requirements. A DL safe rule does not support negation and disjunction operations in the rule.

Tran et al. [31] proposed an approach based on extending Description Logic with one of its sublanguage ALC (Attribute Language Complement) which supports negation and disjunction along with conjunction existential limit and value restriction, which were not supported in earlier approach by $\mathrm{Mu}$. To obtain personalized recommendation ALC (D) is used which represents information in a specific domain. User profile describing items of interest is obtained from the user, which is matched with the resource profile describing all items in the domain to produce personalized recommendations.

Rule based personalization based on extending logical language has advantage in that it has strong ability of expression and decidability but they require intense knowledge of logic programming to write the rules. It is difficult to interpret the rules written in DL by the user thereby abstracting the personalization logic.

\subsubsection{Event Condition Action Rules}

Event-condition-action (ECA) rules support the reactive functionality of Semantic Web. ECA rules allow representing applications reactive functionality to be defined and managed in a single rule base rather than in diverse programs thereby enhancing maintainability and modularity of application [32]. Approaches used for personalization in Semantic Web based on Event Condition Action rules produce recommendations based on the occurrences of events which cause triggering of rule, and the corresponding action is carried if the condition under which action must be carried out is true [33]. The following paragraphs discuss some of the approaches used for rule based personalization using event condition action rules in Semantic Web.

Rule management ontology was created by Debattista et al. [34] for representing rules. Rules are modeled on eventcondition-action pattern concepts in Rule management ontology. Rules are transferred and stored in rule pool.
Context information consisting of set of events are collected and stored as event set in log. Pattern matching is done on rules in rule pool and events in event set. If they match, corresponding actions are carried out to generate personalized recommendation.

Pattern matching is computational expensive and consumes time for computing match. Barla et al. [35] proposed an approach that does not depend on pattern matching to generate personalized recommendations. Instead, it utilizes client and server log information to generate events for a particular user and store it in user model. Events ontology is created describing all events and the attributes associated with the events. Event action rules contain all knowledge about processing $\log$ of events and update the user model. Rules are used to generate personalized recommendations by carrying out mapping between user model and knowledge about previous user preferences.

Approaches used for rule based personalization based on Event-Condition-Action require context information to generate personalized recommendations based on the occurrence of events; they also use information stored in client and server logs to generate recommendations. Collection of context information from various sources is time-consuming. Processing of $\log$ information to find interesting pattern requires time and effort.

\subsubsection{Expert systems}

Expert systems are computer programs used for producing recommendations or problem solving based on knowledge in some domain [36]. Traditional Web-based Expert system does not support the method for representing data in a format that can be used for machine reasoning, which becomes a major drawback for them [37]. This drawback can be overcome by using Semantic Web instead of traditional Web where there is representative format supporting representation of information in a machine process able format. Some of the approaches used for rule-based personalization in Semantic Web using expert systems are discussed in the following paragraphs.

Garcia et al. [38] proposed an approach for producing recommendation based on expert system using fuzzy logic techniques. Customer is the users of this tourist personalization system and is required to submit their preferences information, which is converted into fuzzy sets by an expert describing customer characteristics. Fuzzy rules are evaluated to match the fuzzy representation of hotel with customer characteristics. Results of the match are defuzzified to obtain the concrete results according to the hotel ontology. The best hotel is recommended based on the calculated weights.

García approach did not use a structured storage, such as, an ontological knowledge base to store the information about the domain. Wanner et al. [39] proposed an approach that overcomes this drawback and uses ontology based knowledge base as the main data structure to store information about the environmental domain used for designing an expert system offering personalized support to the citizens in questions related to the environmental conditions in their habitat. Information is collected from the user, environmental data and stored in ontological knowledge base in a uniform format. When user formulate a request regarding environmental conditions personalization is carried out by applying fuzzy reasoning which provides pages related to the current environmental conditions. 
Rule-based personalization approaches based on expert system require fuzzy logic integration with the description logic reasoners to produce recommendations. Integration of fuzzy logic requires transformation of ontology concepts into fuzzy sets which is a cumbersome process

\subsubsection{Rule based Inference Engine}

Rule-based inference engines are always required for inference mechanism based on free form rules. Rule based inference engine when implemented in code, are called reasoners which are software programs used to derive new facts from the existing knowledge [40]. As Semantic Web standards are becoming popular, there is a need for Rulebased inference engine to support intelligent processing of Semantic Web data.

An example of the rule engine based reasoning approach is proposed by Doulaverakis et al. [41] represented as Panacea, which is semantic framework for drug recommendations. Ontologies are used to represent medical knowledge and terminology in Panacea. It uses a layered reasoning approach at first level OWL DL reasoner that is used to resolve the inconsistencies in the ontological representation of medical knowledge at second level RDF Rule reasoner that is used to produce drug recommendations according to the set of medical rules.

\section{CONCLUSION}

Knowledge based recommendation systems using ontologies as the knowledge bases are explored in this paper. Knowledge based recommendation system can be used in any domain. Benefits of applying Knowledge based recommendation system are emphasized in this paper. Rule based personalization system, which is a category of Knowledge based recommendation system is discussed in detail.

\section{REFERENCES}

[1] Berendt, Bettina, Andreas Hotho, Dunja Mladenic, Maarten Van Someren, Myra Spiliopoulou, and Gerd Stumme. "A roadmap for Web mining: From Web to Semantic Web". Springer Berlin Heidelberg, 2004.

[2] Nasraoui, Olfa. "World wide Web personalization." Encyclopedia of Data Mining and Data Warehousing, Idea Group (2005)

[3] Bobadilla, Jesús, Fernando Ortega, Antonio Hernando, and Abraham Gutiérrez. "Recommender systems survey." Knowledge-Based Systems 46 (2013): 109-132.

[4] Gedikli, Fatih, and Dietmar Jannach. "Recommender Systems, Semantic-Based." Encyclopedia of Social Network Analysis and Mining. Springer New York, 2014. 1501-1510.

[5] Felfernig, Alexander, Michael Jeran, Gerald Ninaus, Florian Reinfrank, Stefan Reiterer, and Martin Stettinger. "Basic approaches in recommendation systems." In Recommendation Systems in Software Engineering, pp. 15-37. Springer Berlin Heidelberg, 2014.

[6] Pazzani, Michael J., and Daniel Billsus. "Content-based recommendation systems." In The adaptive Web, pp. 325-341. Springer Berlin Heidelberg, 2007.

[7] Lops, Pasquale, Marco De Gemmis, and Giovanni Semeraro. "Content-based recommender systems: State of the art and trends." In Recommender systems handbook, pp. 73-105. Springer US, 2011.
[8] IJntema, Wouter, Flavius Frasincar, Frederik Hogenboom, and Uzay Kaymak. "News Personalization using the CF-IDF Semantic Recommender." (2011).

[9] Bogdanov, Dmitry, MartíN Haro, Ferdinand Fuhrmann, Anna Xambó, Emilia Gómez, and Perfecto Herrera. "Semantic audio content-based music recommendation and visualization based on user preference examples." Information Processing \& Management 49, no. 1 (2013): 13-33.

[10] Su, Xiaoyuan, and Taghi M. Khoshgoftaar. "A survey of collaborative filtering techniques." Advances in artificial intelligence 2009 (2009): 4.

[11] Aggarwal, Charu C., and S. Yu Philip. "Semantic based collaborative filtering." U.S. Patent 6,487,539, issued November 26, 2002.

[12] Lee Jae-Won, Kim Han-Joon And Lee Sang-Goo, "Applying taxonomic knowledge and semantic collaborative filtering to personalized search: a bayesian belief network based approach",in proceedings of 2010 12th International Asia-Pacific Web Conference IEEE. (2010).

[13] López-Nores, Martín, Yolanda Blanco-Fernández, José J. Pazos-Arias, and Alberto Gil-Solla. "Property-based collaborative filtering for health-aware recommender systems." Expert Systems with Applications 39, no. 8 (2012): 7451-7457.

[14] Fard, Karamollah Bagheri, Mehrbakhsh Nilashi, and Naomie Salim. "Recommender system based on semantic similarity." International Journal of Electrical and Computer Engineering (IJECE) 3, no. 6 (2013): 751 761.

[15] Smyth, Barry. "Case-based recommendation." In The adaptive Web, pp. 342-376. Springer Berlin Heidelberg, 2007.

[16] Daramola, Olawande, Mathew Adigun, and Charles Ayo. "Building an ontology-based framework for tourism recommendation services." Information and Communication Technologies in Tourism 2009 (2009): 135-147.

[17] 17.Felfernig, Alexander, and Robin Burke. "Constraintbased recommender systems: technologies and research issues." In Proceedings of the 10th international conference on Electronic commerce, p. 3. ACM, 2008.

[18] Felfernig, Alexander, Gerhard Friedrich, Dietmar Jannach, and Markus Zanker. "Developing Constraintbased Recommenders." Recommender systems handbook 1 (2011): 187

[19] Felfernig, Alexander, Sarah Haas, Gerald Ninaus, Michael Schwarz, Thomas Ulz, Martin Stettinger, Klaus Isak, Michael Jeran, and Stefan Reiterer. "Recturk: Constraint-based recommendation based on human computation." In RecSys 2014 CrowdRec Workshop, pp 1-6. 2014.

[20] Dell'Aglio, Daniele, Irene Celino, and Dario Cerizza. "Anatomy of a Semantic Web-enabled knowledge-based recommender system." In Proceedings of the 4th international workshop Semantic Matchmaking and Resource Retrieval in the Semantic Web, at the 9th International Semantic Web Conference. 2010. 
[21] Alferes, Jóse Júlio, Carlos Viegas Damásio, and Luís Moniz Pereira. "Semantic Web logic programming tools." In Principles and practice of Semantic Web reasoning, pp. 16-32. Springer Berlin Heidelberg, 2003.

[22] Berners-Lee, Tim, James Hendler, and Ora Lassila. "The Semantic Web." Scientific american 284, no. 5 (2001): 28-37.

[23] Smart, Paul R. "Rule-Based Intelligence on the Semantic Web: Implications for Military Capabilities." (2007)

[24] Antoniou, Grigoris, Matteo Baldoni, Cristina Baroglio , Robert Baumgartner, François Bry, Thomas Eiter, Nicola Henze et al." Reasoning methods for personalization on the Semantic Web". na, 2004.

[25] Antoniou, G., Billington, D., Governatori, G., And Maher, M." Representation Results for Defeasible Logic". ACM Transactions on Computational Logic 2,2 (2002), 255-287.

[26] Heymans, S., And Vermeir, D. "Integrating Semantic Web reasoning and answer set programming". In Answer Set Programming, Advances in Theory and Implementation, Proc. 2nd Intl. ASP'03 Workshop, Messina, Italy (2003), pp. 194-208

[27] Alferes, José Júlio, Antonio Brogi, João Alexandre Leite, and Luís Moniz Pereira. "Evolving logic programs." In Logics in Artificial Intelligence, pp. 50-62. Springer Berlin Heidelberg, 2002.

[28] Mu, Xiangwei, Yan Chen, Yan Cao, and Yan Lin. "Personalized Recommendation System Modeling in Semantic Web." Advances in Information Sciences and Service Sciences 5, no. 2 (2013): 278.

[29] Baader, Franz, Ian Horrocks, and Ulrike Sattler. "Description logics as ontology languages for the Semantic Web." In Mechanizing Mathematical Reasoning, pp. 228-248. Springer Berlin Heidelberg, 2005

[30] Krötzsch, Markus, Frantisek Simancik, and Ian Horrocks. "Description Logics."IEEE Intelligent Systems 29, no. 1 (2014): 12-19.

[31] Tran, Thanh, Philipp Cimiano, and Anupriya Ankolekar. "A Rule-Based Adaption Model for Ontology-Based Personalization." In Advances in Semantic Media Adaptation and Personalization, pp. 117-135. Springer Berlin Heidelberg, 2008.
[32] Papamarkos, George, Alexandra Poulovassilis, and Peter T. Wood. "Event-Condition-Action Rule Languages for the Semantic Web." In SWDB, pp. 309-327. 2003.

[33] Moore, Philip T., and Hai V. Pham. "Personalization and rule strategies in data-intensive intelligent context-aware systems." The Knowledge Engineering Review 30, no. 02 (2015): 140-156.

[34] Debattista, Jeremy, Simon Scerri, Ismael Rivera, and Siegfried Handschuh. "Ontology-based Rules for Recommender Systems." In SeRSy, pp. 49-60. 2012.

[35] Barla, Michal, Michal Tvarožek, and Mária Bieliková. "Rule-based user characteristics acquisition from logs with semantics for personalized Web-based systems." Computing and Informatics 28, no. 4 (2012): 399-428.

[36] P. Jackson, "Introduction to Expert Systems", 3rd edition. Boston: Addison- Wesley Longman Publishing Co., 2001

[37] Verhodubs, Olegs, and Janis Grundspenkis. "Towards the Semantic Web Expert System." Scientific Journal of Riga Technical University. Computer Sciences 44, no. 1 (2011): 116-123.

[38] García-Crespo, Ángel, José Luis López-Cuadrado, Ricardo Colomo-Palacios, Israel González-Carrasco, and Belén Ruiz-Mezcua. "Sem-Fit: A semantic based expert system to provide recommendations in the tourism domain."Expert systems with applications 38, no. 10 (2011): 13310-13319.

[39] Wanner, Leo, Marco Rospocher, Stefanos Vrochidis, Lasse Johansson, Nadjet Bouayad-Agha, Gerard Casamayor, Ari Karppinen et al. "Ontology-centered environmental information delivery for personalized decision support." Expert Systems with Applications 42, no. 12 (2015): 5032-5046.

[40] Singh, Swapna, and Ragini Karwayun. "A comparative study of inference engines." In Information Technology: New Generations (ITNG), 2010 Seventh International Conference on, pp. 53-57. IEEE, 2010.

[41] Doulaverakis, Charalampos, George Nikolaidis, Athanasios Kleontas, and Ioannis Kompatsiaris. "Panacea, a semantic-enabled drug recommendations discovery framework." In VDOS+ DO@ ICBO. 2013. 